\title{
Bose-Einstein correlations and color reconnection in hadronic W
}

\author{
Raimund Ströhmer ${ }^{* \dagger}$ \\ LMU Muenchen, Germany \\ E-mail: Raimund.StroehmerePhysik.Uni-Muenchen.de
}

\begin{abstract}
We report on studies of Bose-Einstein correlations and color reconnection in hadronic $\mathrm{W}$ pair production at LEP. Bose-Einstein correlations between identical particles from the decay of different W's are studied by comparing the particle correlation in hadronic W pair decays to those in events which are constructed from the combination of the hadronic parts of two events where one of the Ws decays leptonicaly. The LEP combined result of the strength of the effect is consistent with zero and can be used to limit the systematic uncertainty due to Bose-Einstein correlations on the W mass measurements.

Color reconnection is expected to affect the production of particles in hadronic decays of W pairs. Measurements of inclusive charged particle multiplicities, and of their angular distribution with respect to the four jet axes of the events, are used to test models of color reconnection. The results are both consistent with models without color reconnection and models with moderate color reconnection. They can be used to exclude more extreme models of color reconnection and thereby limit the uncertainty due to color reconnection on the $\mathrm{W}$ mass measurement.
\end{abstract}

International Europhysics Conference on High Energy Physics

July 21st - 27th 2005

Lisboa, Portugal

\footnotetext{
*Speaker.

${ }^{\dagger}$ For the LEP Collaborations.
} 


\section{Introduction}

In $\mathrm{W}$ pair events both $\mathrm{W}$ bosons can decay hadronically. In this case two strongly interacting color singlet objects with considerable space time overlap are produced. It is interesting to study whether the the two $\mathrm{W}$ bosons then decay independently or whether they influence each other. In addition to the principle interest in this question possible interactions and correlations between the decay product of the two $\mathrm{W}$ bosons influence the precision measurement of the $\mathrm{W}$ mass at LEP and are treated in the mass measurement as source of systematic uncertainty.

\section{Bose-Einstein correlations}

Bose-Einstein correlations (BEC) between identical bosons are a well-known phenomenon in high energy physics [1]. BEC lead to an enhancement of the production of identical bosons close in phase space. At LEP BEC have been unambiguously established between the particles originating from one hadronically decaying $\mathrm{W}$, representing so-called intra-W BEC [2]. The possible additional BEC between the particles originating from different $\mathrm{W}$ bosons, i.e. inter-WW BEC, has to be separated from the intra-W BEC. BEC are usually presented in terms of two-particle densities, $\rho_{2}(Q)$, measured as

$$
\rho_{2}(Q)=\frac{1}{N_{\text {events }}} \frac{\mathrm{d} N_{\text {pairs }}}{\mathrm{d} Q}
$$

for the number $N_{\text {pairs }}$ of pairs of identical bosons with four-momenta $p_{1}$ and $p_{2}$ and $Q=\sqrt{-\left(p_{1}-p_{2}\right)^{2}}$ in the number $N_{\text {events }}$ of events under study.

The observed two-particle densities in the $\mathrm{W}$ pair decays is then compared with the expectation in the case of non inter-WW BEC which is constructed from $\mathrm{W}$ pairs where only one $\mathrm{W}$ decays as:

$$
2 \rho_{2}^{\mathrm{W}}(Q)+2 \rho_{\text {mix }}^{\mathrm{WW}}(Q) .
$$

The second term is a measure of the two-particle densities between particles from different $\mathrm{W}$ bosons which decay independently. Is is estimated by artificially mixing the hadronic part of two semileptonic $\mathrm{W}$ pair events. Care has to be taken that existing correlations between the two W bosons are represented in the mixed events. Only events which polar angles pointing to the same detector region (e.g. $\left|\theta_{\mathrm{W}^{+}}-\theta_{\mathrm{W}^{-}}\right| \leq 75 \mathrm{mrad}$ or $\left|\left(\pi-\theta_{\mathrm{W}^{+}}\right)-\theta_{\mathrm{W}^{-}}\right| \leq 75 \mathrm{mrad}$ ) are combined and one event is then rotated so that both event are back to back. The standard selection for hadronic W pair selection is then applied to the mixed events. Monte Carlo simulations and distributions not sensitive to BEC are used to establish that these mixed events represent uncorrelated $\mathrm{W}$ pair events. In order to maximize the sensitivity and to minimize systematic uncertainties different ways to compare the measured two-particle density with that estimated for events without interWW BEC have been used.

$$
\Delta \rho(Q)=\rho_{2}^{\mathrm{WW}}(Q)-2 \rho_{2}^{\mathrm{W}}(Q)-2 \rho_{\text {mix }}^{\mathrm{WW}}(Q), \quad \delta_{I}(Q)=\Delta \rho(Q) / \rho_{\text {mix }}^{\mathrm{WW}}(Q), \quad D(Q)=\frac{\rho_{2}^{\mathrm{WW}}(Q)}{2 \rho_{2}^{\mathrm{W}}(Q)+2 \rho_{\text {mix }}^{\mathrm{WW}}(Q)}
$$

By either comparing the result for the data with the expectation from Monte Carlo without BEC or by comparing the result from same-sign pairs with opposite-sign pairs uncertainties due to the mixing procedure can be reduced.

$$
\Delta \rho^{\prime}(Q)=\Delta \rho(Q)-=\Delta \rho_{\mathrm{no}-\operatorname{BECMC}}(Q), \quad D^{\prime}(Q)=\frac{D(Q)}{D_{\text {no-BECMC }}(Q)}
$$




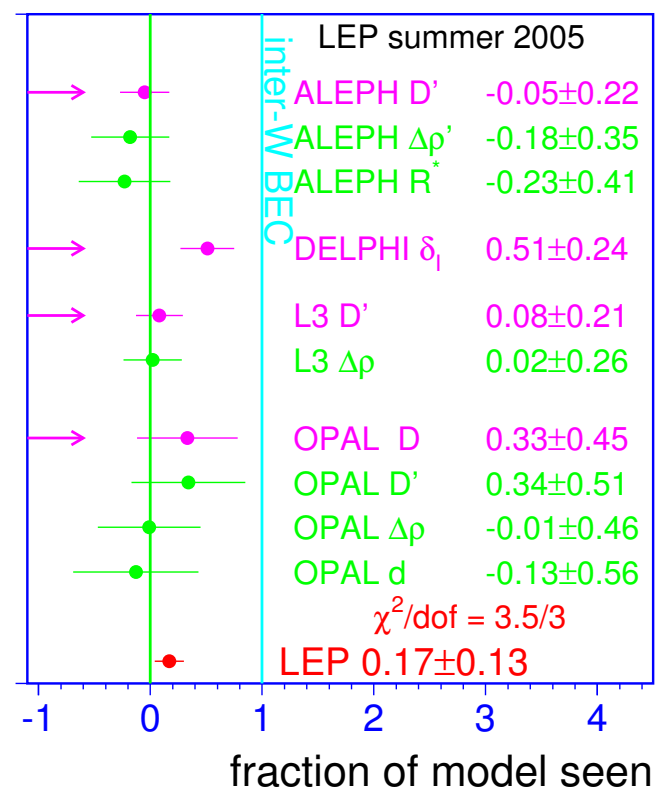

Figure 1: Relative strength of inter-W BEC compared to the strength of intra-W BEC. The measurements marked with arrows are used for the combination.

$$
d(Q)=D( \pm \pm) / D(+-) . \quad R^{*}=\left(N_{\pi}^{++,--} / N_{\pi}^{+-}\right)^{\text {data }} /\left(N_{\pi}^{++,--} / N_{\pi}^{+-}\right)_{\text {no-BEC }}^{M C}
$$

The measured distributions are then compared to Monte Carlo simulations with inter-W BEC correlation. The strength of that correlation relative to the strength of intra-W BEC is then varied to determine which scaling factor best describes the measurements. Results for different observables from the four LEP experiments together with a combination are shown in Figure 1[3]. No significant evidence for inter-W BEC correlation was found.

\section{Color Reconnection}

The decay products of the two $\mathrm{W}$ bosons in hadronic $\mathrm{W}$ pair decays have a significant spacetime overlap as the separation of their decay vertices at LEP2 energies is small compared to characteristic hadronisation distance scales. It is therefore feasible that colored objects from both W bosons interact during the hadronisation. With the current understanding of non-perturbative QCD, such interference can be estimated only in the context of specific models $[4,5,6]$. In general this models predict a changed charged multiplicity and a changed particle flow in the regions between jets from different $\mathrm{W}$ bosons (inter-W) compared to the regions between jets from the same $\mathrm{W}$ boson (intra-W). The comparison of charged multiplicity in hadronic $\mathrm{W}$ pair decays to that in semiletonic $\mathrm{W}$ pair decays is unfortunately not precise enough to constrain the color reconnection models significantly. The definition of regions between different jets in 4 -jet events is non-trivial and slightly different approaches have been used by the LEP experiments. Figure 2 (left) shows a comparison of the ratio of particle flow in the inter- to the intra-W regions between the data and different Monte Carlo Models without and with color reconnection[7]. In the SK-I model[4] the strength of the color reconnection can be adjusted. One can clearly see that the extreme scenario 

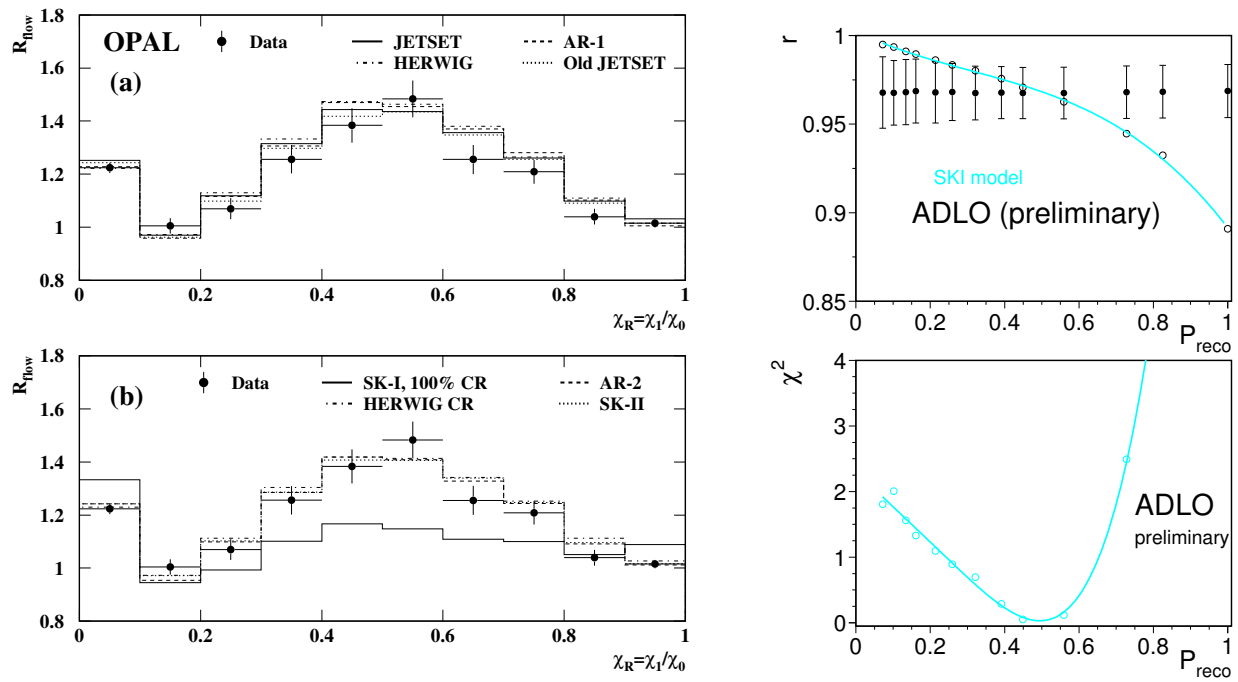

Figure 2: Left: Comparison of the ratio of particle flow in the inter to the intra $\mathrm{W}$ regions between the data and different Monte Carlo Models without (top) and with (bottom) color reconnection. Right: Constraint on the strength of color reconnection from the LEP combination

of $100 \% \mathrm{CR}$ (which is used as a benchmark and is not considered as a realistic scenario by the authors) is excluded. The measurement can be used to determine the most likely strength of color reconnection in the SK I model. The result of the LEP combination is shown in Figure 2 on the right[3].

\section{References}

[1] E.A. De Wolf, I.M. Dremin, W. Kittel, Phys. Rep. 270 (1996) 1;

I.G. Knowles, G.D. Lafferty, J. Phys. G 23 (1997) 731.

[2] DELPHI Collaboration, J. Abdallah et al., Eur. Phys. J. C 44 (2005) 161;

OPAL Collaboration, G. Abbiendi et al., Eur. Phys. J. C 36 (2004) 297;

ALEPH Collaboration, R. Barate et al., Phys. Lett. B 478 (2000) 50;

ALEPH Collaboration, S. Schael et al., Phys. Lett. B 606 (2005) 265;

L3 Collaboration, P. Achard et al., Phys. Lett. B 547 (2002) 139.

[3] The LEP Collaborations, hep-ex/0511027

[4] T. Sjöstrand and V.A. Khoze, Z. Phys. C62 (1994) 281; Phys. Rev. Lett. 72 (1994) 28.

T. Sjöstrand, Comput. Phys. Commun. 135 (2001) 238.

[5] L. Lönnblad, Z. Phys. C70 (1996) 107.

L. Lönnblad, Comput. Phys. Commun. 71 (1992) 15.

[6] Proceedings of CERN LEP2 Workshop, CERN 96-01, Vol. 2, p. 159, eds. G. Altarelli, T. Sjöstrand and F. Zwirner, February 1996.

G. Corcella et al., J. High Energy Phys. JHEP 01 (2001) 010.

[7] OPAL Collaboration, G. Abbiendi et al., Submitted to Eur.Phys.J.C hep-ex/0508062 